\title{
Design of Band-Gap Structures from Sheet Metal
}

\author{
D. IWAŃSKI* AND J. WICIAK \\ Department of Mechanics and Vibroacoustics, Faculty of Mechanical Engineering and Robotics \\ AGH University of Science and Technology, al. A. Mickiewicza 30, 30-059 Kraków, Poland
}

\begin{abstract}
Two-dimensional, circular plate was taken under consideration to design a band-gap structure. The analytical model was built using the Bessel functions and at the end the numerical examples were presented using MatLab and Ansys software. Results show that it is possible to create a band-gap structure, however further verification analysis must be made to improve given solution.
\end{abstract}

PACS: $46.40 .-\mathrm{f}$

\section{Introduction}

The propagation and reduction of waves in mechanical systems is a classical problem in structural dynamics [1]. When high performance is needed, active methods of control can be used $[2,3]$. However such methods are often complicated and expensive. Thus, the common approach to mitigate sound and vibration is to use passive methods. One of such technique is based on well-known physical phenomenon that periodic structures have several band gaps in the frequency domain, where no wave propagation is possible [4]. These band-gaps can be easily obtained in one-dimensional structures (beams) by adding masses to such structures [5]. The beam is infinitely long (authors [5] also examined real finite beam supported at the ends) and additional elements must have an equal geometry, weight and distance among them. Figure 1 shows a sample of described structure.

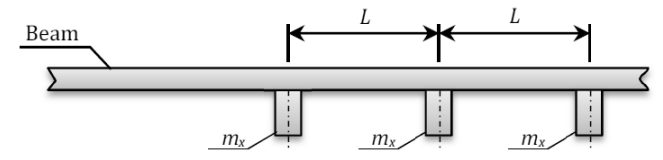

Fig. 1. A finite beam with periodically attached masses; $L_{x}$ - distance between each masses $\left(m_{x}\right)$.

If such structure is exposed to external force then the frequency spectrum of the vibration of the structure will contain band-gaps. Frequency band-gaps in such structure mainly depend on two factors: value of mass and distance among them. In other work [7] author developed analytical solution for vibration of two-dimensional structure - a circular Mindlin plate with concentric elastic ring supports. At the end some interesting numerical results were presented.

* corresponding author; e-mail: iwanski@agh.edu.pl
The aim of this work is to extend Sorokin and Ershova's work [5] where one-dimensional beam was used for calculations, to two-dimensional plate. This may give more opportunity in application because the geometry of larger structure (two-dimensional) could provide reduction of the noise on larger area. As opposed to the Xiang work [7] circular plate is not supported on the rings but on the border of the plate. Number of two elastic rings was chosen after Sorokin and Ershova [5] and for the source force of vibration there was taken a point force with constant amplitude transversal along circumference and located in the centre of the plate.

The analytical solution was built and some numerical results of insertion loss were presented. Finally the finite element model of described structure was built in order to determine the eigenfrequencies of the structure. Finally these frequencies were compared with negative values of insertion loss.

\section{Problem statement}

Considering an isotropic thin elastic plate, an example of which is shown in Fig. 2, some assumptions must be made:

- geometry of the plate is circular;

- the plate is thin elastic and infinitely long [5];

- two rings compose additional masses, which increase mass of the plate.

If the plate has finite length then at the end of the plate there would be a dissipation of energy at the fixing point. Infinitely long plate avoids this problem [5]. Circular shape provides symmetry and gives results at every point of designed structure in radial $(r)$ and circumferential $(\varphi)$ coordinates. Rings are symmetrical along both axes. They have the same material properties but different geometry and mass. These assumptions simplify the calculations. 


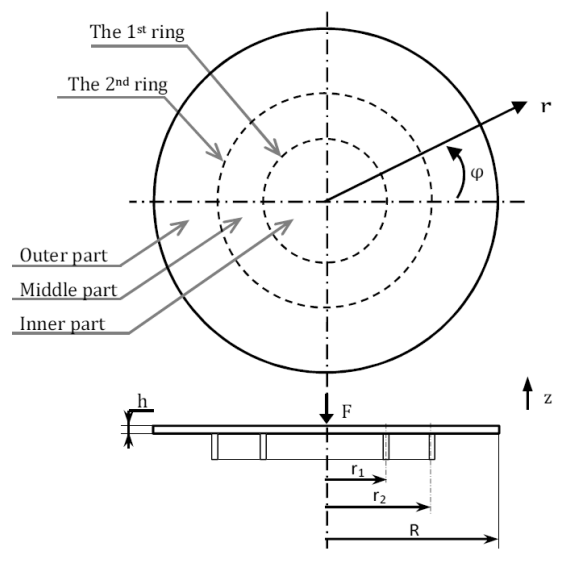

Fig. 2. Circular plate with two rings attached in cylindrical coordinate system $(r, \varphi, z) ; r_{1}, r_{2}$ - radius of the first and second ring, respectively, $R$ - radius of the plate, $h$ - thickness of the plate, $F$ - external point force.

In order to solve this problem mathematically, the plate model has been divided into three parts: inner bounded by the first ring, middle - bounded by the first and the second ring and outer — bounded by the second ring and ended in infinitive. For the numerical calculations the radius of the outer part, $R$, needs to have fixed length.

\section{Approximate analytical solution}

\subsection{Model}

Plate model has been located in polar coordinate system $r, \varphi$ in the median plane and the centre of the plate intersects the median plane as the origin. The deflection of the plate $w$ is small with respect to its thickness $h$, which is small with respect to the radius of the plate $R$.

First, free oscillations of the plate were taken under consideration. The boundary conditions for this statement is that for $r=R$ dynamic deflection of the plate $W=0$ and the slope $\partial W / \partial r=0$. The differential equation of deflection of the plate has the following form [8]:

$$
D \nabla^{2} \nabla^{2} W+k W+\gamma h \frac{\partial^{2} W}{\partial t^{2}}-I_{2} \frac{\partial^{2}}{\partial t^{2}}\left(\nabla^{2} W\right)=0,
$$

where $I_{2}=\gamma h / 12$ is the rotatory inertia, $D$ - flexural rigidity, $k-$ stiffness, and $\gamma$ is the density. When free vibration is assumed, the deflection is periodic and can be expressed as

$$
W(r, \varphi, t)=w(r, \varphi) \cos \omega t,
$$

where $w$ - angular frequency of the oscillations. Substituting Eq. (2) into Eq. (1) becomes

$$
D \nabla^{2} \nabla^{2} w+k w-\gamma h \omega^{2} w+I_{2} \omega^{2} \nabla^{2} w=0 .
$$

In further calculations a simplified version of Eq. (3) was taken under considerations, which has the form [6]

$$
\left(\nabla^{4}-\lambda^{4}\right) w=0
$$

where

$$
\lambda^{4}=\frac{\gamma h \omega^{2}-k}{D} .
$$

Next considerations were made after passing from variable $r$ to the dimensionless quantity $\xi=\lambda r$ (called reduced distance).

The general solution of Eq. (4) is

$$
\begin{aligned}
& w(\xi, \varphi)=\sum_{n=0}^{\infty}\left[A_{n} J_{n}(\xi)+B_{n} Y_{n}(\xi)+C_{n} I_{n}(\xi)\right. \\
& \left.+D_{n} K_{n}(\xi)\right] \cos n \varphi+\sum_{n=1}^{\infty}\left[A_{n}^{*} J_{n}(\xi)+B_{n}^{*} Y_{n}(\xi)\right. \\
& \left.\quad+C_{n}^{*} I_{n}(\xi)+D_{n}^{*} K_{n}(\xi)\right] \sin n \varphi,
\end{aligned}
$$

where $J_{n}, Y_{n}, I_{n}$ and $K_{n}$ are the cyllindrical Bessel functions. The coefficients $A_{n}, B_{n}, C_{n}$ and $D_{n}$, which determine the mode shapes, are solved using boundary conditions.

According to Fig. 2 and the distribution of the plate into inner, middle and outer part, three equations of the deflection must be solved, one for each part, respectively. Denoting the deflection of these parts with $w_{\text {in }}, w_{\mathrm{m}}$ and $w_{\text {out }}$ and assuming the $n$-th term for this case to be 0 then for the middle part of the plate Eq. (6) becomes

$$
w_{\mathrm{m}}(\xi)=C_{1} J_{0}(\xi)+C_{2} Y_{0}(\xi)+C_{3} I_{0}(\xi)+C_{4} K_{0}(\xi)(7)
$$
and the outer part

$$
w_{\text {out }}(\xi)=C_{5} H_{0}^{(1)}(\xi)+C_{6} K_{0}(\xi),
$$

where $H_{\nu}(z)$ - the Bessel cyllindrical function. For the inner part more calculations must be made. The general solution for this part will have the following form:

$$
w_{\text {in }}(\xi)=w_{0}(\xi)+w_{\mathrm{F}}(\xi),
$$

where $w_{\mathrm{F}}$ and $w_{0}$ are the deflection of the plate with and without the concentrated load at the centre, respectively.

If the plate is solid and there is no point force at the centre of the plate, then the deflection at $\xi=0$ should be finite. The expansion of the functions $Y_{0}(\xi)$ and $K_{0}(\xi)$ imply that these functions are infinite as $\xi \rightarrow 0$. However, this fact does not imply that $C_{3}=0$ and $C_{4}=0$. In the vicinity of the point $\xi=0$, these functions can be represented in the following form [9]:

$$
\begin{aligned}
& Y_{0}(\xi)=\frac{2}{\pi}\left(1-\frac{\xi^{2}}{4}\right) \ln \xi+\frac{2 \gamma}{\pi}+\ldots, \\
& K_{0}(\xi)=-\left(1+\frac{\xi^{2}}{4}\right) \ln \xi-\gamma+\ldots
\end{aligned}
$$

The omitted terms and their derivatives which appear in the result of the fulfilled differentiation tend to zero as $\xi \rightarrow 0$. Hence, after omitting these terms, the results of the calculation will not be changed.

Setting $C_{4}=2 C_{3} / \pi$ the sum of the second and the fourth terms in Eq. (7) will have the form

$$
w_{\mathrm{F}}(\xi)=C_{3}\left[Y_{0}(\xi)+\frac{2}{\pi} K_{0}(\xi)\right] \text {. }
$$


In order to find the transversal force along a circumference of a small radius $\xi$, calculation of the sum of the transversal force along this circumference and passing it to the limit as $\xi \rightarrow 0$ must be made. The conditions of the equilibrium of this part of the plate which is bounded by the circumference of the radius $\xi / \lambda$, imply that this limit is equal to the concentrated load $\boldsymbol{F}$ (Fig. 2), acting on the plate, taken with the opposite sign. Thus, in order to determine the constant, expression (12) must be introduced into the following equation:

$$
\lim _{\xi \rightarrow 0}\left[-D \lambda^{3} \frac{\mathrm{d}}{\mathrm{d} \xi}\left(\frac{\mathrm{d}^{2} w}{\mathrm{~d} \xi^{2}}+\frac{1}{\xi} \frac{\mathrm{d} w}{\mathrm{~d} \xi}\right)\right] \frac{2}{\pi} \frac{\xi}{\lambda}=-F,
$$

where $F$ - external force. In this calculation the following relations will be used [9]:

$$
\begin{aligned}
& \frac{\mathrm{d}^{2} Y_{0}(\xi)}{\mathrm{d} \xi^{2}}+\frac{1}{\xi} \frac{\mathrm{d} Y_{0}(\xi)}{\mathrm{d} \xi}=-Y_{0}(\xi), \\
& \frac{\mathrm{d}^{2} K_{0}(\xi)}{\mathrm{d} \xi^{2}}+\frac{1}{\xi} \frac{\mathrm{d} K_{0}(\xi)}{\mathrm{d} \xi}=K_{0}(\xi) .
\end{aligned}
$$

After introducing the expression (12) into Eq. (13) and using Eqs. (14) and (15) it will have the form

$$
\lim _{\xi \rightarrow 0}\left[-D C_{3} \lambda^{2}\left(-Y_{0}^{\prime}(\xi)+\frac{2}{\pi} K_{0}^{\prime}(\xi)\right) 2 \pi \xi\right]=-F .
$$

Using the expressions (10) and (11), after differentiating and passing to the limit, for the determination of $C_{3}$ it results in the relation $-8 D C_{3} \lambda^{2}=F$, hence $C_{3}=-F /\left(8 D \lambda^{2}\right)$. Thus, Eq. (12) can be written in the following form:

$$
w_{\mathrm{F}}(\xi)=-\frac{F}{8 D \lambda^{2}\left[Y_{0}(\xi)+2 K_{0}(\xi) / \pi\right]} .
$$

If there is no point force at the centre the terms involving $Y_{n}$ and $K_{n}$ in the solution (6) must be discarded in order to avoid infinitive values at the origin, $r=0$.

\section{Hence it becomes}

$$
w_{0}(\xi)=A_{n} J_{0}(\xi)+C_{n} I_{0}(\xi) .
$$

Introducing Eqs. (17) and (18) into Eq. (9) and rewriting the coefficients $A_{n} \ldots C_{n}$ the equations for the inner part $w_{\text {in }}$, middle part $w_{\mathrm{m}}$ and the outer part $w_{\text {out }}$ of the plate have the following form:

$$
\begin{aligned}
& w_{\text {in }}(\xi)=C_{1} J_{0}(\xi)+C_{2} I_{0}(\xi) \\
& -\frac{F}{8 D \lambda^{2}\left[Y_{0}(\xi)+\frac{2}{\pi} K_{0}(\xi)\right]} \\
& w_{\mathrm{m}}(\xi)=C_{3} J_{0}(\xi)+C_{4} Y_{0}(\xi)+C_{5} I_{0}(\xi)+C_{6} K_{0}(\xi), \\
& w_{\text {out }}(\xi)=C_{7} H_{0}^{(1)}(\xi)+C_{8} K_{0}(\xi) .
\end{aligned}
$$

Equations (19), (20) and (21) contain coefficients $C_{1}, C_{2}, \ldots, C_{8}$ which will be solved using boundary conditions.

\subsection{Boundary conditions}

From the description of the model it is clear that the deflection and the slope of the inner and middle part of the plate must be the same and equal to deflection and slope of the first ring, respectively. The similar solution is found for the middle and outer plate and the second ring. Their deflections and slopes must remain equal.

Denoting the deflection of the rings by $w_{r 1}$ and $w_{r 2}$, and the slopes of the rings by $\mathrm{d} w_{r 1} / \mathrm{d} \xi$ and $\mathrm{d} w_{r 2} / \mathrm{d} \xi$ respectively, the boundary conditions will have the form for $\xi=r_{1}\left(r_{1}\right.$ - radius of the 1 st ring $)$ :

$$
\begin{aligned}
& w_{\text {in }}(\xi)=w_{\mathrm{m}}(\xi)=w_{r 1}(\xi), \\
& \frac{\mathrm{d}}{\xi} w_{\mathrm{m}}(\xi)=\frac{\mathrm{d}}{\mathrm{d} \xi} w(\xi)=\frac{\mathrm{d}}{\mathrm{d} \xi} w_{r 1}(\xi) ;
\end{aligned}
$$

for $\xi=r_{2}\left(r_{2}\right.$ - radius of the 2 nd ring $)$ :

$$
\begin{aligned}
& w_{\mathrm{m}}(\xi)=w_{\text {out }}(\xi)=w_{r 2}(\xi), \\
& \frac{\mathrm{d}}{\xi} w_{\mathrm{m}}(\xi)=\frac{\mathrm{d}}{\mathrm{d} \xi} w_{\text {out }}(\xi)=\frac{\mathrm{d}}{\mathrm{d} \xi} w_{r 2}(\xi) .
\end{aligned}
$$

The deflections $w_{r 1}, w_{r 2}$ and the slopes $\mathrm{d} w_{r 1} / \mathrm{d} \xi$, $\mathrm{d} w_{r 2} / \mathrm{d} \xi$ will be solved using equations of equilibrium of the forces and moments (Fig. 3), taking cross-section of symmetrical plate as a beam.

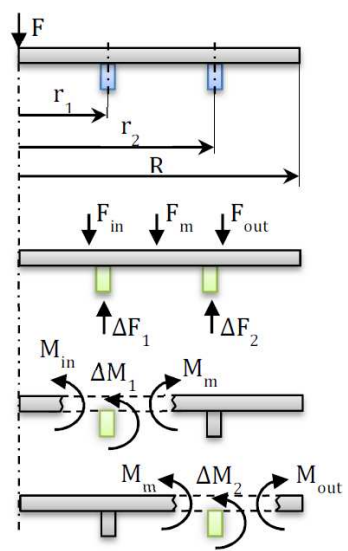

Fig. 3. Forces and moments in analyzed model.

Equations of equilibrium of the forces and moments (Fig. 3):

$$
\begin{aligned}
& \Delta F_{1}=F_{\text {in }}+F_{\mathrm{m}}, \\
& \Delta F_{2}=F_{\mathrm{m}}+F_{\text {out }}, \\
& \Delta M_{1}=M_{\text {in }}-M_{\mathrm{m}}, \\
& \Delta M_{2}=M_{\mathrm{m}}-M_{\text {out }} .
\end{aligned}
$$

Expressing Eq. (23) with deflection of the ring yields

$$
F_{\text {in }}+F_{\mathrm{m}}=w_{r 1} m_{1} \omega^{2}-w_{r 1} k_{1}=w_{r 1}\left(m_{r 1} \omega^{2}-k_{1}\right),
$$

hence 


$$
w_{r 1}=\frac{F_{\mathrm{in}}+F_{\mathrm{m}}}{m_{r 1} \omega^{2}-k_{1}},
$$

where $m_{r 1}$ and $k_{1}$ are the mass and rigidity of the first ring, respectively. Similarly Eq. (24) becomes

$$
F_{\mathrm{m}}+F_{\text {out }}=w_{r 2}\left(m_{r 2} \omega^{2}-k_{2}\right),
$$

hence

$$
w_{r 2}=\frac{F_{\mathrm{m}}+F_{\text {out }}}{m_{r 2} \omega^{2}-k_{2}},
$$

where $m_{r 2}$ and $k_{2}$ are the mass and rigidity of the second ring respectively.

Due to inertial forces in the rings under external load, Eqs. (25) and (26) have the form [10]:

$$
\begin{gathered}
M_{\mathrm{in}}-M_{\mathrm{m}}=I_{p 1} \omega^{2} \frac{\mathrm{d}}{\mathrm{d} \xi} w_{r 1}-k_{1 \mathrm{~T}} \frac{\mathrm{d}}{\mathrm{d} \xi} w_{r 1}+\frac{E I_{1}}{r_{1}^{2}} \frac{\mathrm{d} w_{r 1}}{\mathrm{~d} \xi} \\
=\frac{\mathrm{d}}{\mathrm{d} \xi} w_{r 1}\left(I_{p 1} \omega^{2}-k_{1 \mathrm{~T}}+\frac{E I_{1}}{r_{1}^{2}}\right),
\end{gathered}
$$

hence

$$
\begin{aligned}
& \frac{\mathrm{d}}{\mathrm{d} \xi} w_{r 1}=\frac{M_{\mathrm{in}}-M_{\mathrm{m}}}{I_{p 1} \omega^{2}-k_{1 \mathrm{~T}}+\frac{E I_{1}}{r_{1}^{2}}}, \\
& M_{\mathrm{m}}-M_{\text {out }}=\frac{\mathrm{d}}{\mathrm{d} \xi} w_{r 2}\left(I_{p 2} \omega^{2}-k_{2 \mathrm{~T}}+\frac{E I_{2}}{r_{2}^{2}}\right),
\end{aligned}
$$

hence

$$
\frac{\mathrm{d}}{\mathrm{d} \xi} w_{r 2}=\frac{M_{\mathrm{m}}-M_{\mathrm{out}}}{I_{p 2} \omega^{2}-k_{2 \mathrm{~T}}+\frac{E I_{2}}{r_{2}^{2}}} .
$$

$E$ - Young's modulus, $I$ - second area moment of interia, $I_{p}$ - second moment of interia, $k_{\mathrm{T}}$ - torsional stiffness.

Equations of symmetrical bending force and moments of the plate have the following form [11]:

$$
\begin{aligned}
& F_{n}=-D\left(\frac{\mathrm{d}^{3} w_{n}}{\mathrm{~d} r^{3}}+\frac{1}{r} \frac{\mathrm{d}^{2} w_{n}}{\mathrm{~d} r^{2}}-\frac{1}{r^{2}} \frac{\mathrm{d} w_{n}}{\mathrm{~d} r}\right), \\
& M_{n}=-D\left(\frac{\mathrm{d}^{2} w_{n}}{\mathrm{~d} r^{2}}+\frac{\nu}{r} \frac{\mathrm{d} w_{n}}{\mathrm{~d} r}\right),
\end{aligned}
$$

where $w_{n}$ is the deflection in the $n$-th part of the plate and $\nu$ - the Poisson ratio. Substituting Eq. (31) in expressions (27) and (28) with appropriate deflection $w_{n}$ and Eq. (32) in expressions (29) and (30) yields to define deflections $\left(w_{r 1}, w_{r 2}\right)$ and slopes $\left(\mathrm{d} w_{r 1} / \mathrm{d} \xi, \mathrm{d} w_{r 2} / \mathrm{d} \xi\right)$ of the rings.

Above calculations yield to use boundary conditions to calculate unknown parameters $C_{1}, C_{2}, \ldots, C_{8}$. Having these parameters the deflection for each part of the plate can be obtained.

In order to find out how the rings affect frequency band an insertion loss (IL) described by Eq. (33) was used to calculate the difference between the deflections of the plate with $\left(w_{\text {in }}\right)$ and without rings $\left(w_{\mathrm{nr}}\right)$ :

$$
\mathrm{IL}=20 \log \frac{I\left[w_{\mathrm{nr}}(0)\right]}{I\left[w_{\mathrm{in}}(0)\right]} .
$$

To do so, another equation must be solved. Equation (34) presents the deflection of the plate without any rings

$$
w_{\mathrm{nr}}(\xi)=C_{9} H_{0}^{(1)}+C_{10} K_{0}(\xi) .
$$

Using boundary condition that the slope $\mathrm{d} w / \mathrm{d} r$ at the $r=0$ must be equal to 0 the unknown parameters $C_{9}$ and $C_{10}$ equal

$$
C_{9}=-\frac{F}{\mathrm{i} 4 D \lambda^{2}(1+\pi)}, \quad C_{10}=\mathrm{i} 4 C_{1} .
$$

Substituting these parameters to Eq. (34) and canceling the Bessel functions $Y$ and $K$ (they cancel each other) the equation for deflection of the plate without rings in the point where the load is applied has the following form:

$$
w_{\mathrm{nr}}(0)=-\frac{F}{\mathrm{i} 4 D \lambda^{2}(1+\pi)} \text {. }
$$

\section{Numerical results}

Analytical solution for given problem was calculated numerically using MatLab software. Each of two plots present calculated insertion loss for sample values of the parameters of the rings. The constants here (besides the material constants which is linear and elastic steel) are radius of the plate $R=0.5 \mathrm{~m}$, its thickness $h=0.001 \mathrm{~m}$, external force $F=20 \mathrm{~N}$, mass of the first ring $m_{1}=$ $1 \mathrm{~kg}$ and for the second ring four values of masses $m_{2}=$ $\{0.5,1,2,5\} \mathrm{kg}$. Geometry parameters of the rings (width equal to height) were automatically calculated from the moment of inertia for each ring.

Next step was to compare given results with eigenfrequencies of the structure.

\section{Conclusions}

In this work analytical model of two-dimensional, circular plate was built, described using the Bessel functions and the numerical examples were presented using MatLab and Ansys software. From these results the following conclusions can be made:

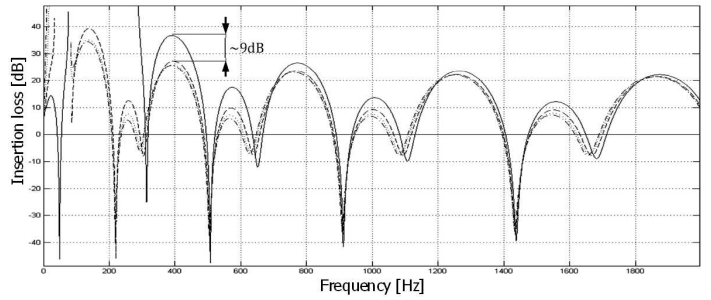

Fig. 4. Insertion loss for $r_{1}=0.2 \mathrm{~m}$ and $r_{2}=0.4 \mathrm{~m}$.

- there are significant differences in insertion loss between plate with and without additional rings (masses) when coaxial force is applied (Figs. 4, 5); 


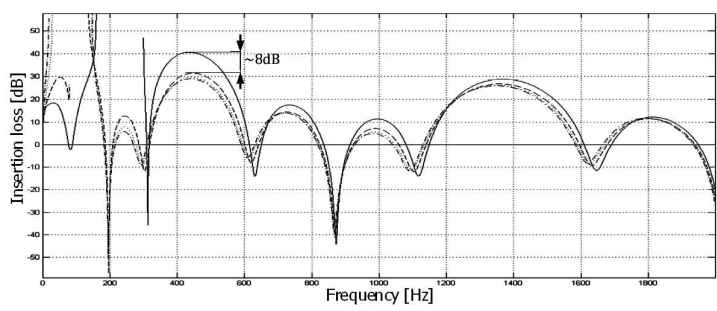

Fig. 5. Insertion loss for $r_{1}=0.1 \mathrm{~m}$ and $r_{2}=0.3 \mathrm{~m}$.

- as expected there are frequency ranges where the band-gap is present, however there are frequencies for which amplification can be observed. This corresponds to eigenfrequencies of additional masses and their harmonics, solved using finite element model (Tables I, II);

- there are significant changes between the values of insertion loss for different masses used as second ring. The higher value of mass of second ring in the smaller insertion loss is observed and the frequency range is lower.

\section{TABLE I}

Eigenfrequencies calculated using Ansys and MatLab software for given rings parameters: $m_{1}=1 \mathrm{~kg}, m_{2}=0.5 \mathrm{~kg}$, $r_{1}=0.2 \mathrm{~m}, r_{2}=0.4 \mathrm{~m}$

\begin{tabular}{c|c|c}
\hline \hline \multirow{2}{*}{ Mode } & \multicolumn{2}{|c}{ Frequency $[\mathrm{Hz}]$} \\
\cline { 2 - 3 } & Ansys & MatLab \\
\hline 35 & 308 & 313 \\
56 & 507 & 507 \\
70 & 646 & 652 \\
96 & 916 & 912 \\
149 & 1412 & 1437 \\
168 & 1683 & 1683
\end{tabular}

TABLE II

Eigenfrequencies calculated using Ansys and MatLab software for given rings parameters: $m_{1}=1 \mathrm{~kg}, m_{2}=1 \mathrm{~kg}, r_{1}=$ $0.1 \mathrm{~m}, r_{2}=0.3 \mathrm{~m}$.

\begin{tabular}{c|c|c}
\hline \hline \multirow{2}{*}{ Mode } & \multicolumn{2}{|c}{ Frequency $[\mathrm{Hz}]$} \\
\cline { 2 - 3 } & Ansys & MatLab \\
\hline 18 & 197 & 196 \\
33 & 314 & 304 \\
64 & 631 & 620 \\
86 & 855 & 869 \\
105 & 1099 & 1104 \\
146 & 1622 & 1634
\end{tabular}

Numerical examples present only a brief view of calculations of investigated model. A further work must be done in order to improve numerical analysis.

\section{Acknowledgments}

The author wishes to acknowledge the consistent guidance and encouragement of Professor S.V. Sorokin of Aalborg University.

\section{References}

[1] L. Cremer, M. Heckl, B.A.T. Petersson, Structure-Borne Sound. Structural Vibrations and Sound Radiation at Audio Frequencies, 3rd ed., Springer, Berlin 2005.

[2] A. Preumont, K. Seto, Active Control of Structures, Wiley, United Kingdom 2008.

[3] J. Wiciak, Mol. Quant. Acoust. 25, 281 (2004).

[4] D.J. Mead, J. Sound Vibr. 190, 495 (1996).

[5] S.V. Sorokin, O.A. Ershova, J. Sound Vibrat. 278, 501 (2004).

[6] A.W. Leissa, Vibration of Plates, NASA 1973, Washington (D.C, USA).

[7] Y. Xiang, Int. J. Mech. Sci. 45, 497 (2003).

[8] J.N. Reddy, Theory and Analysis of Elastic Plates and Shell, 2nd ed., Taylor \& Francis Group, New York 2007.

[9] B.G. Korenev, Bessel Functions and Their Applications, Taylor \& Francis Group, London 2002.

[10] A.E.H. Love, A Treatise on the Mathematical Theory of Elasticity, 4th ed., NY Dover Publications, New York 1944.

[11] S. Timoshenko, S. Woinowsky-Krieger, Theory of Plates and Shells, McGraw-Hill Book Co., USA 1959. 